\title{
Sarcomere assembly in C. elegans muscle*
}

\author{
Donald G. Moerman ${ }^{\S}$, Department of Zoology, University of British \\ Columbia, Vancouver, B.C., Canada V6T 1 Z4 \\ Benjamin D. Williams ${ }^{\S}$, Department of Cell and Structural Biology, \\ University of Illinois, Urbana, IL, 61801 USA
}

\section{Table of Contents}

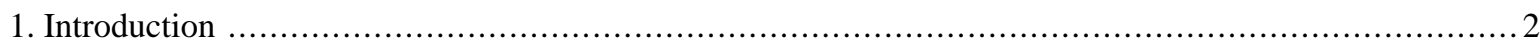

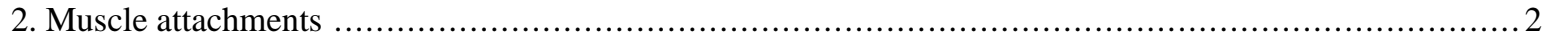

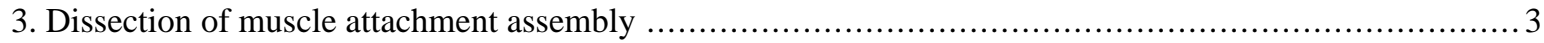

4. Initiating a sarcomere leads to distinct assembly dependence pathways for dense bodies and M-lines ....... 4

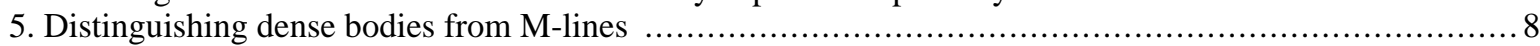

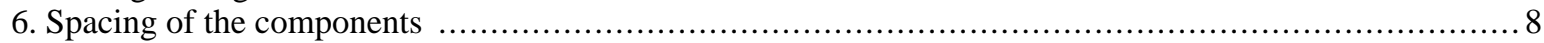

7. Is sarcomere assembly in $C$. elegans a general model of sarcomere assembly? ........................ 12

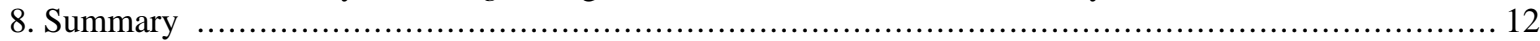

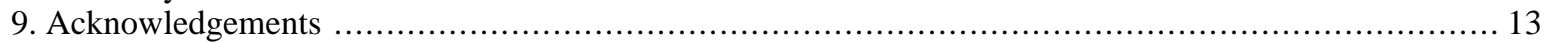

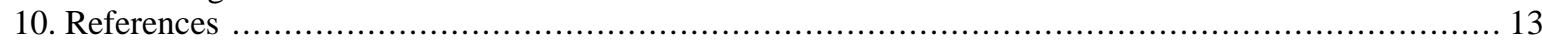

\begin{abstract}
Sarcomeres within body wall muscle in $C$. elegans include attachments to the sarcolemma that are remarkably similar in structure to vertebrate adhesion complexes. Crucial early steps in muscle sarcomere assembly, a highly orchestrated affair involving many proteins, involve the assembly of these sarcomere attachments. The steps involved in initiating the correct placement of these attachments and other sarcomere substructures are poorly understood. Using mutants in $C$. elegans we are attempting to dissect the various steps in this process. We review what has been discovered to date and present a model of sarcomere assembly that initiates at the plasma membrane and involves proteins within muscle, the hypodermis and within the extracellular matrix.
\end{abstract}

\footnotetext{
*Edited by James M. Kramer and Donald G. Moerman. Last revised May 5, 2005. Published January 16, 2006. This chapter should be cited as: Moerman, D. G. and Williams, B. D. Sarcomere assembly in C. elegans muscle (January 16, 2006), WormBook, ed. The C. elegans Research Community, WormBook, doi/10.1895/wormbook.1.81.1, http://www.wormbook.org.

Copyright: (C) 2006 Donald G. Moerman and Benjamin D. Williams. This is an open-access article distributed under the terms of the Creative Commons Attribution License, which permits unrestricted use, distribution, and reproduction in any medium, provided the original author and source are credited.

${ }^{\S}$ To whom correspondence should be addressed. E-mail: moerman@zoology.ubc.ca or bdwillms@life.uiuc.edu
} 


\section{Introduction}

The nematode Caenorhabditis elegans has proven to be a useful system to study the development of muscle (Waterston, 1988; Moerman and Fire, 1997). Here we review sarcomere assembly, focusing specifically on the early events that occur at the muscle cell membrane. Myoblasts arise after the end of gastrulation (at 290 min. of embryonic development; Sulston et al., 1983) and are defined by the accumulation of structural components such as myosin, actin, vinculin, and integrin (Epstein et al., 1993; Hresko et al., 1994). Myoblasts then migrate to their final positions in four longitudinal quadrants, each of which is a double row of muscle cells (Hresko et al., 1994; Schnabel et al., 1997). The myoblasts flatten basally against the hypodermis and laterally against the neighboring muscle cell. The result is a continuous muscle-muscle junction running down the center of the muscle quadrant that is flanked on either side by a muscle-hypodermal junction (Hresko et al., 1994). In mid embryogenesis (450 min), the sarcomere components move to the muscle cell membrane where the muscle-muscle and muscle-hypoderm junctions converge at the center of the muscle quadrant, and then organize into sarcomeres. At this same time they form attachment structures that provide a physical linkage laterally between muscles cells and basally between muscle and hypodermis (Hresko et al., 1994).

\section{Muscle attachments}

There are three related attachment complexes formed, the muscle-muscle adhesion plaques, and two related muscle-hypodermal structures termed dense bodies and M-lines. Although the attachment plaques also contribute, the dense bodies and M-line are primarily responsible for translating the mechanical movement of myofibrillar components to motion of the whole animal (Francis and Waterston, 1985).

In adult muscle cells both dense bodies and M-lines are finger-like projections that extend from the muscle cell membrane into the cytoplasm where they ultimately attach to and organize the myofilaments within the contractile units. This arrangement is illustrated in Figure 1, which shows the overall distribution of C. elegans body wall muscle and the organization of sarcomeres within muscle. Dense bodies and M-lines attach to actin filaments and myosin filaments, respectively, and are homologs of the Z-lines and M-lines in vertebrate striated muscle. The attachment plaques at muscle-muscle junctions are most similar to dense bodies, and anchor the actin filaments of the last half-I-band to the cell membrane (Francis and Waterston, 1985).
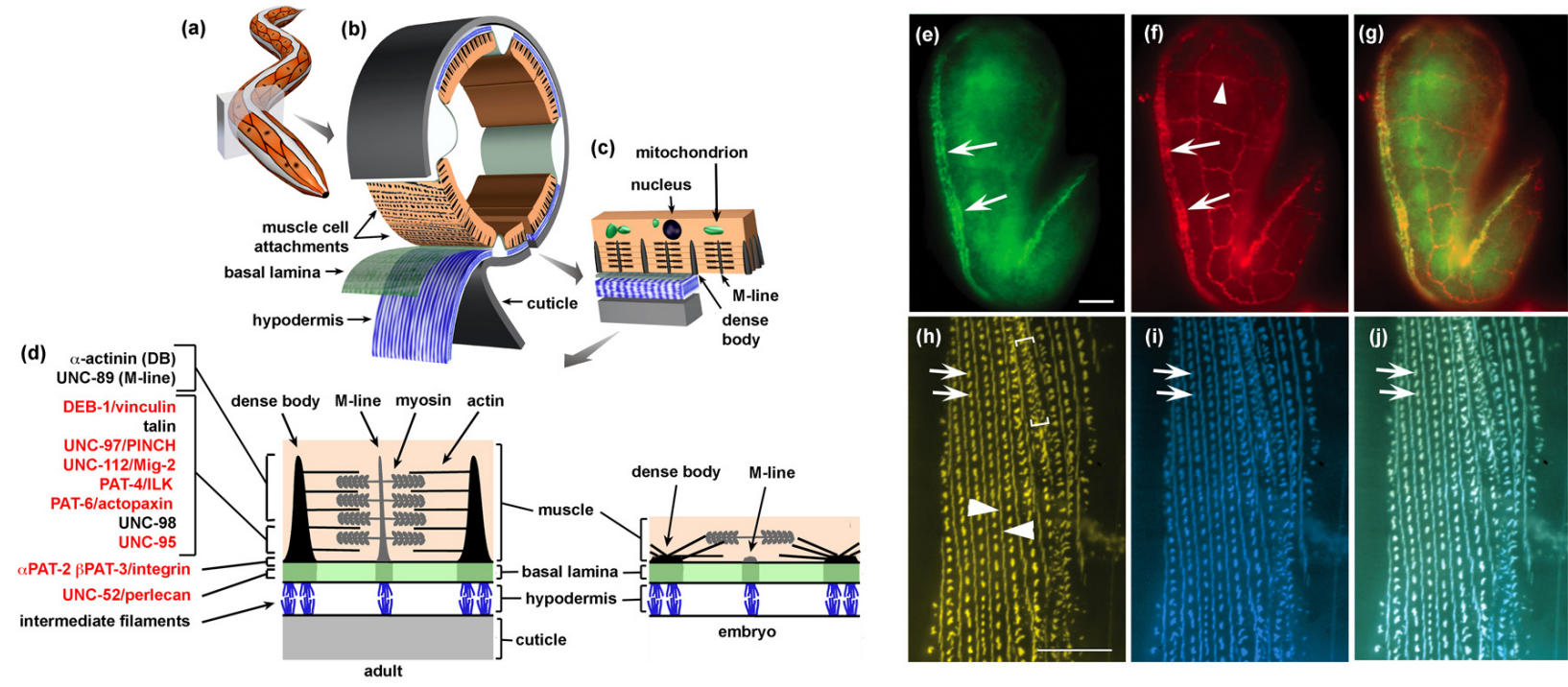

Figure 1. Schematic Diagram of the $C$. elegans Body-Wall Muscle Structure, and fluorescence microcopy of muscle attachments in embryos and adults. (A) An adult worm with body-wall muscle quadrants visible (orange). (B) A body-wall cross-section with cuticle, hypodermis, and basal lamina peeled away to reveal the basal membrane of two body-wall muscle cells. (C) A longitudinal section through a body-wall muscle cell. Dense bodies and M-lines attach actin thin filaments and myosin thick filaments, respectively, to the basal sarcolemma. (D) Locations of several different muscle attachment proteins. Loss-of-function for proteins shown in red causes the Pat developmental arrest phenotype. (E-J). PAT-4/ILK and PAT-3 integrin colocalize at muscle attachments in embryos and adults. (E) A wild-type, 420 min. embryo. PAT-4::GFP localizes to body-wall muscle attachments (arrow). The scale bar represents $2 \mu \mathrm{m}$. (F) The same embryo as that shown in (E) double stained with monoclonal antibodies MH25, recognizing PAT-3 integrin, and MH27, recognizing hypodermal-hypodermal cell junctions (included for developmental staging and orientation purposes). (G) An overlay of (E) and (F). Areas of PAT-4::GFP and integrin colocalization appear yellow. (H-J) Detail of body-wall muscle from a rescued pat-4(st551) adult hermaphrodite coexpressing (H) pat-4::yfp and (E) pat-3::cfp. Dense bodies (arrows), M-lines (arrowheads), and muscle-muscle adhesion plaques (brackets in H) are indicated. (J) An overlay of panels $(\mathrm{H})$ and (I). Regions in which PAT-3::CFP and PAT-4::YFP colocalize appear white. The scale bar in (H) represents $5 \mu$ m. Right-click or control-click for larger image. 
Here we discuss early events in the assembly of muscle attachments, so it is important to recognize that all three types of attachments appear as morphologically similar dense plaques when they are first formed in embryos (Hresko et al., 1994). This is indicated for dense bodies and M-lines on the right of panel d in Figure 1. It is only during larval stages that the dense bodies and M-lines extend deeper into the cytoplasm and assume the shape of finger-like projections from the membrane (Francis and Waterston, 1985). Interestingly, at least for the dense body, projection from the membrane corresponds with the addition of the membrane-distal component, alpha-actinin (Francis and Waterston, 1985; Barstead and Waterston, 1991).

\section{Dissection of muscle attachment assembly}

The extracellular matrix (ECM) receptor PAT-2/PAT-3 integrin (Gettner et al., 1995; B. Williams, unpublished results) is concentrated at the membrane of attachment plaques, as well as dense bodies and M-lines, allowing them to transmit mechanical force across the muscle cell membrane to the basal lamina. This force can be transmitted, either between adjacent muscle cells, or between muscle and hypodermis. Fibrous organelle (FO's) complexes in the hypodermis, which are similar to hemi-desmosomes, then complete the mechanical linkage to the cuticular exoskeleton (Francis and Waterston, 1991). Patterning of the muscle to hypodermis linkage may involve the hypodermal transmembrane protein myotactin at the hypodermal/muscle interface (Hresko et al., 1999). A major component of the hemidesmosome at hypodermal/muscle and hypodermal/cuticle interfaces is VAB-10/ spectroplakin (Bosher et al., 2003). Also probably present at both sites is the VAB-19/Krank protein (Ding et al., 2003). The linkage from the epidermis to the cuticle is through cytoplasmic intermediate filaments (e.g., тиа-6; Hapiak et al., 2003). Present only at the hypodermal/cuticle interface are the transmembrane proteins, MUA-3 and MUP-4 (Bercher et al., 2001; Hong et al., 2001), which mediate the final step in the mechanical linkage to the cuticle.

Just as occurs in vertebrate integrin-mediated attachments between the ECM and the actin cytoskeleton, dense bodies contain cytoskeletal adaptor proteins such as vinculin, alpha-actinin, talin, PINCH, MIG-2, integrin linked kinase (ILK), and actopaxin, which complete the linkage between the cytoplasmic domain of integrin and actin filaments (see Figure 2; Francis and Waterston, 1985; Barstead and Waterston, 1991; Moulder et al., 1996, Hobert et al., 1999, Rogalski et al., 2000; Mackinnon et al., 2002; Lin et al., 2003). The M-lines contain many of the same membrane-proximal adaptors, but lack vinculin. The membrane-distal region of the M-line lacks the dense body protein alpha-actinin, but does include the M-line specific protein UNC-89 (Benian et al., 1996). Given their protein composition and functions, dense bodies and M-lines are both analogous and homologous to vertebrate integrin mediated adhesion plaques, commonly called focal adhesions (FA's) in tissue culture cells (Figure 2; for review see Burridge and Chrzanowska-Wodnicka, 1996; and Geiger and Bershadsky, 2001). In effect, these attachment complexes and associated myofilaments can be thought of as a highly ordered set of focal adhesions that offer a unique opportunity for genetic analysis. The regulatory steps that coordinate the assembly of adherens junction components into functional attachment structures capable of enduring and transmitting mechanical stress are largely undefined. Genetic screens for mutants with defects in sarcomere assembly have proven a powerful aid in identifying novel focal adhesion proteins and for the investigation of their functions in vivo (for example, see Williams and Waterston, 1994). Mutants with early, severe defects in sarcomere assembly fail to begin normal embryonic movements at mid-embryogenesis, and soon after this point the morphogenetic process of elongation stops prematurely at the two fold length. This is referred to as the Pat developmental arrest phenotype (Paralyzed, Arrested elongation at Two fold). 


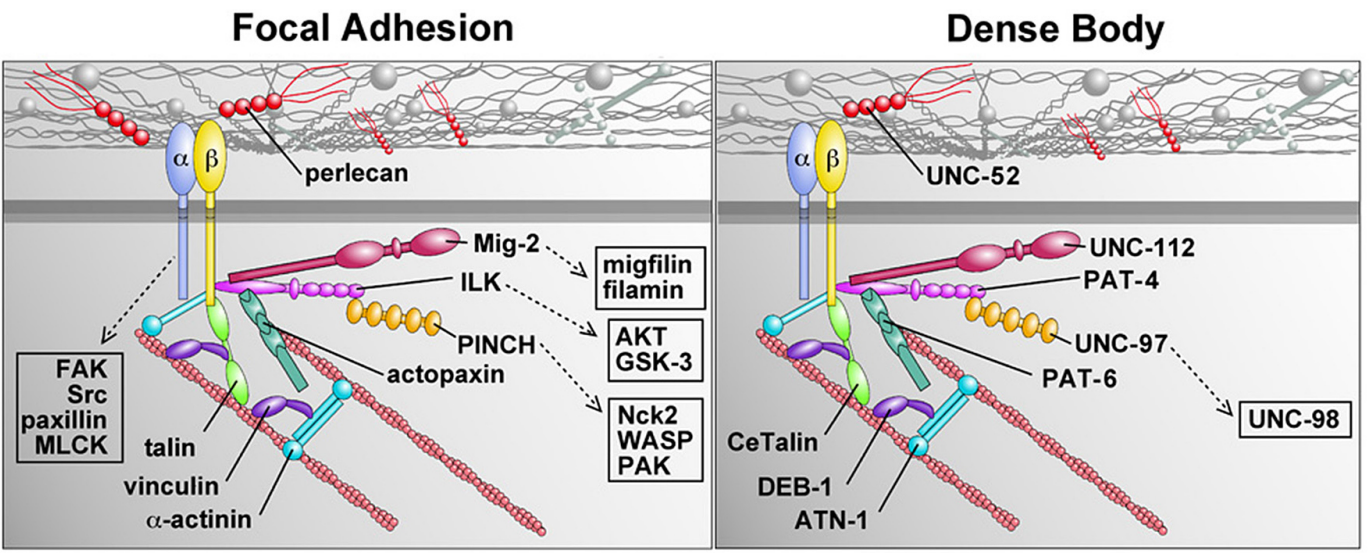

Figure 2. Comparison between vertebrate focal adhesions and C. elegans dense bodies. Modified from Figure 5 in Cox and Hardin (2004). It should be noted in the dense body diagram that PAT-4/ILK is shown making contact with the cytoplasmic domain of the PAT-3 integrin subunit because this interaction has been reported for the vertebrate homologs. To date we have failed to observe this binding interaction in $C$. elegans, so the nature of any linkage between PAT-3 integrin and the complex of proteins that bind to PAT-4 remains to be elucidated Similarly, the potential interactions between PAT-3 integrin, CeTalin, alpha-actinin and DEB-1/vinculin are based upon the binding interactions demonstrated for the vertebrate homologs. See text for details.

\section{Initiating a sarcomere leads to distinct assembly dependence pathways for dense bodies and M-lines}

Genetic dissection of muscle attachment assembly has allowed us to construct distinct "assembly dependence" pathways for dense body and M-lines (see Table 1 and Figure 3). These pathways differ in their membrane-distal components, probably reflecting the separate roles of dense bodies and M-lines in anchoring either thin or thick filaments to the cell membrane. Within each pathway, location of one protein above another with an intervening arrow means that the top protein is recruited to attachments independent of the protein below, or any of the other proteins further down the pathway. Reciprocally, the recruitment of the protein below is dependent upon the protein above. This diagram therefore shows the results of a genetic epistasis analysis of "assembly dependence". It is important to note that the arrows indicate specific requirements for protein recruitment in vivo, rather than evidence of direct protein-protein binding in teractions. Proteins within parallel branches in the pathway are recruited independent of one another to attachments. Conversely, two proteins located at the same level in the pathway (i.e., not separated by an arrow) are mutually dependent on each other for recruitment.

\section{A}

\section{DENSE BODY}

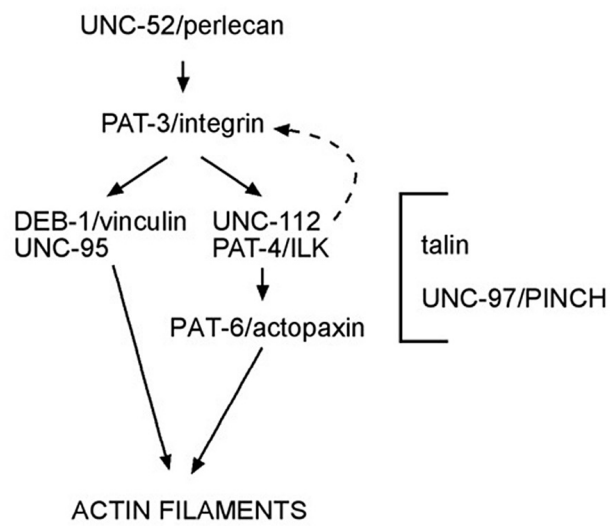

B

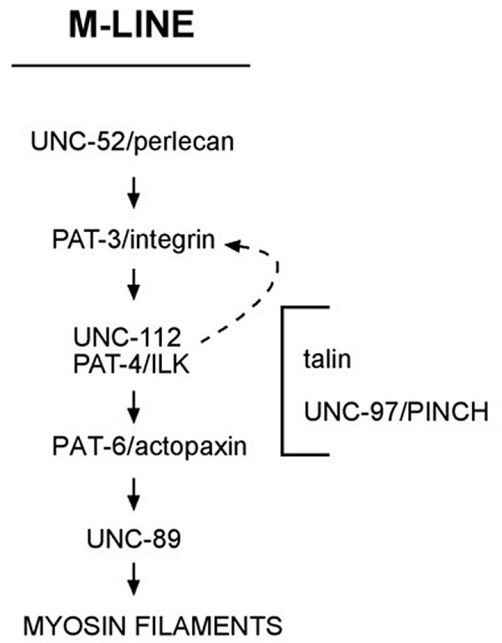

Figure 3. "Assembly dependence" pathways for dense bodies and M-lines. See text for details. 
Table 1. Muscle cell phenotype in Pat mutants

\begin{tabular}{|c|c|c|c|}
\hline Genes & Cell polarization & $\begin{array}{c}\text { Myosin } \\
\text { organization }\end{array}$ & $\begin{array}{c}\text { Actin } \\
\text { organization }\end{array}$ \\
\hline $\begin{array}{l}\text { Class I } \\
\text { unc-52 (perlecan) } \\
\text { pat-2 (alpha-integrin) } \\
\text { pat-3 (beta-integrin) }\end{array}$ & No & No & No \\
\hline $\begin{array}{l}\text { Class II } \\
\text { pat-34 (ILK) } \\
\text { unc-97 (PINCH) } \\
\text { unc-112 (MIG-2) } \\
\text { pat-6 (Actopaxin) } \\
\text { pat-11 }\end{array}$ & Yes & No & No \\
\hline $\begin{array}{l}\text { Class III } \\
\text { deb-1 (vinculin) } \\
\text { pat-9 } \\
\text { pat-12 }\end{array}$ & Yes & Yes & Yes \\
\hline $\begin{array}{l}\text { Class IV } \\
\text { lev-11 (Tropomyosin) } \\
\text { egl-19 (L-type calcium channel) } \\
\text { tnc-1 (troponin C) } \\
\text { mup-2 (troponin T) }\end{array}$ & Yes & Yes & Yes \\
\hline $\begin{array}{l}\text { Class V } \\
\text { myo-3 (myosin heavy chain) }\end{array}$ & Yes & No & Yes \\
\hline
\end{tabular}

Modified from Williams and Waterston (1994).

We have also included one "counter current" dashed arrow in each of the pathways. These arrows indicate more subtle requirements for proper attachment assembly than gross recruitment, as we describe in more detail below. The first protein to consider is UNC-52/perlecan, which is found in the basement membrane between the body wall muscle cells and the hypodermis, and is concentrated at muscle cell dense bodies and M-lines (Francis and Waterston, 1991; Rogalski et al., 1993; Mullen et al., 1999). UNC-52/perlcan is at the top of each pathway because protein-null mutations in the unc-52 gene block all subsequent steps in sarcomere development, including the next step, which is the polarization of integrin to the basal membrane and its subsequent aggregation, (for each of the four muscle quadrants) into a stripe of small, isotropically arranged focal contacts (Figure 1E-G). At this stage, UNC-52 is normally organized in a corresponding stripe within the adjacent basal lamina. (Rogalski et al., 1993; Hresko et al., 1994; Williams and Waterston, 1994; Mullen et al., 1999). The interaction of integrin and perlecan, either directly or indirectly, is a key early event in the assembly of these attachment structures. While there is no evidence in C. elegans for a direct interaction of UNC-52 and integrin there is evidence for a direct protein-protein interaction between integrin and perlecan in vertebrates (Hayashi et al., 1992; Chakravarti et al., 1995; Brown et al., 1997). The PAT-2/PAT-3 integrin heterodimer is next in the pathway because removal of integrin does not block UNC-52/perlecan deposition in the basal lamina, but does block the recruitment of all other attachment proteins and myofilaments to the muscle cell membrane (Rogalski et al., 1993; Hresko et al., 1994; Williams and Waterston, 1994).

During subsequent steps of normal attachment assembly, other components are recruited to the focal contacts, and as the nascent complexes mature, they enter into a highly ordered array of recognizable dense bodies and M-lines. We have shown that the recruitment of UNC-112/Mig-2 (Rogalski et al., 2000) and PAT-4/ILK 
(Mackinnon et al., 2002) are mutually dependent, but that both are required for the recruitment of PAT-6/actopaxin (Lin et al., 2003). The three proteins form a complex, UNC-112/Mig-2 and PAT-6/actopaxin binding to the amino portion of PAT-4/ILK (Figure 4). Interestingly, we have found that PAT-6 protein is not detectable in pat-4 mutants, suggesting that the stability of PAT-6/actopaxin may be dependent upon its entry into a complex with PAT-4/ILK (Lin et al., 2003).

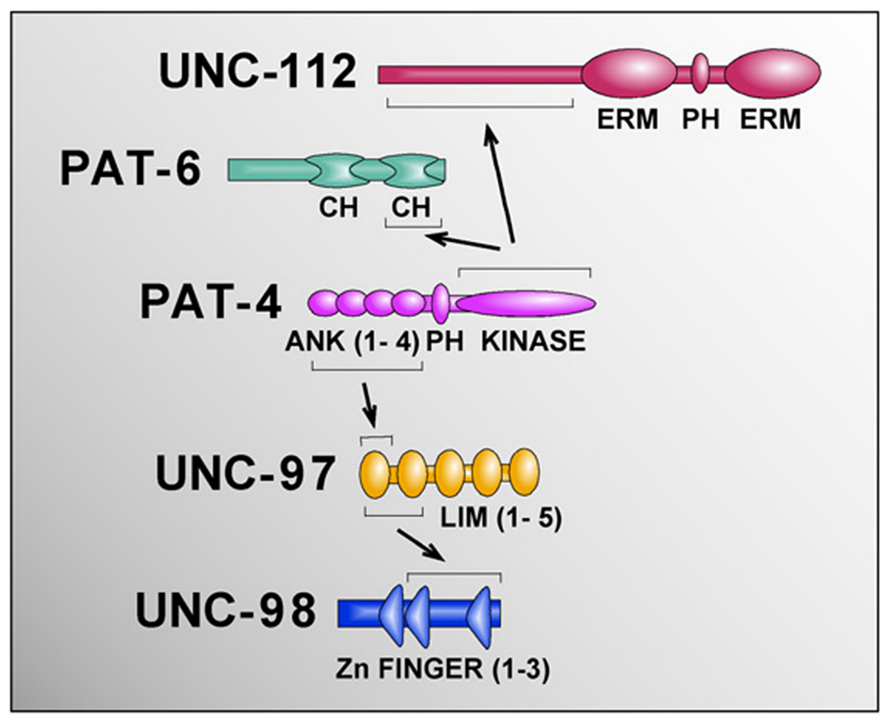

Figure 4. Interactions between UNC-112, UNC-97, PAT-4, PAT-6 and UNC-98 as determined by yeast two-hybrid analysis.

DEB-1/vinculin is placed in a parallel branch because its recruitment to attachments is independent of UNC-112, PAT-4 and PAT-6, and reciprocally, the recruitment of UNC-112, PAT-4 and PAT-6 is not dependent upon DEB-1/vinculin (Rogalski et al., 2000; Mackinnon et al., 2002; Lin et al., 2003). This feature may reflect vinculin's involvement in the separate "classical" linkage between integrin and the cytoskeleton involving talin and $\alpha$-actinin (illustrated in Figure 5). Actin filaments are placed at the convergence of the UNC-112, PAT-4, PAT-6 and the DEB-1 branches because removal of any of these four proteins blocks the normal recruitment of actin filaments to the basal membrane. Similarly, myosin thick filaments are at the bottom of the M-line pathway because removal of any of the upstream components blocks thick filament recruitment to the cell membrane.

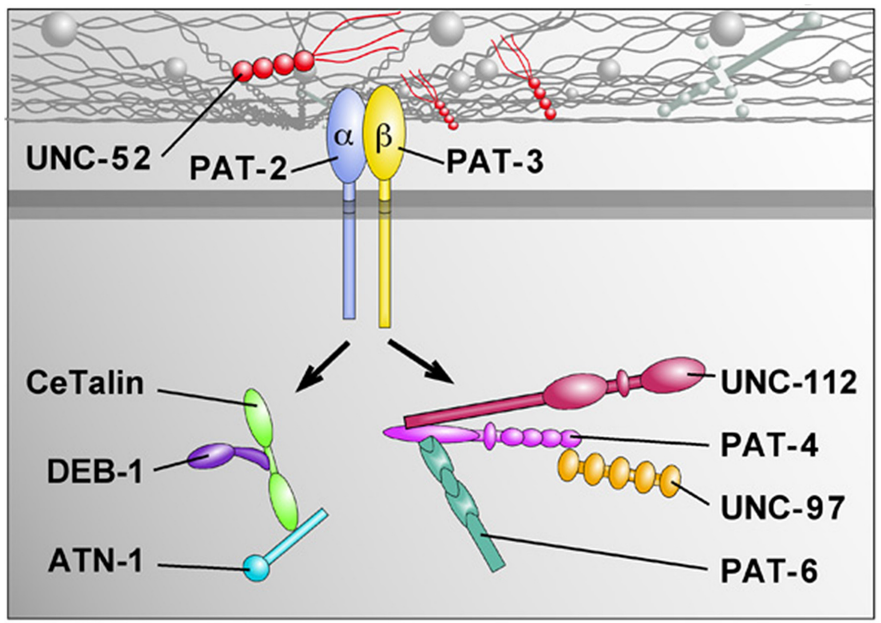

Figure 5. Two different linkage pathways between integrin and actin filaments. (Left) The classic talin linkage, including talin, vinculin and alpha-actinin. (Right) Linkage through the PAT-4/ILK complex.

To this point we have indicated the assembly dependencies between proteins as revealed by substantial disruptions in protein recruitment to nascent attachments. Our genetic analysis has also revealed additional dependencies that are subtler, but nevertheless crucial for proper attachment formation. Although the removal of UNC-112, PAT-4 or PAT-6 does not affect integrin's polarization to the basal membrane and entering nascent focal contacts, removing any of these members of the PAT- 4 complex does disrupt the refinement of integrin patterning 
within the cell membrane. In these mutants integrin never enters the recognizable striated arrays that correspond to dense bodies and M-lines (Rogalski et al., 2000; Mackinnon et al., 2002; Lin et al., 2003). We indicate this more subtle type of assembly dependence with a dashed arrow (Figure 3) in both the dense body and M-line pathways that extends from the PAT-4 complex back to integrin. The relationships indicated by these counter-current arrows are perhaps not surprising. The complexity of these muscle attachments seem almost certain to have many such interdependencies that will preclude a simple linear pathway of assembly.

At least three LIM domain proteins are also involved in adhesion complexes in C. elegans, UNC-95, UNC-97/PINCH and UNC-98 (Broday et al., 2004; Hobert et al., 1999, Norman et al., unpublished; Mercer et al., 2003). Of these three genes only unc-97 has a Pat null phenotype suggesting that like unc-112, pat-4 and pat-6, it is involved with the very early stages of dense body and M-line organization (Hobert et al., 1999; Norman et al., unpublished). Indeed, in unc-97, unc-112, pat-4 and pat-6 mutants, integrin and vinculin are recruited to nascent attachments but myofilaments are not recruited, and the stalled complexes never form a striated array. Although additional experiments are needed to place UNC-97 relative to UNC-112, PAT-4 and PAT-6 on the assembly dependence pathway, the biochemical data support the notion that these proteins work together during attachment assembly. Similar to mammalian PINCH (Tu et al., 1999), UNC-97 binds to the carboxy portion of PAT-4/ILK and is found in dense bodes and M-lines (Norman et al., unpublished; Hobert et al., 1999; Figure 4).

Null alleles of unc-95 and unc-98 affect myofilament organization and both proteins are located in dense bodies and M-lines (Broday et al., 2004; Mercer et al., 2003). UNC-95 is first detected in dense bodes and is only later found in M-lines as well, while UNC-98 is found initially in M-lines and only sporadically in dense bodies (there is some disagreement here between GFP fusion and antibody results). UNC-98 has been shown to bind to UNC-97/PINCH, but no binding studies have yet been done with UNC-95 and members of the PAT-4/ILK complex. Curiously, vinculin levels within the dense body are dependent on UNC-95; loss of UNC-95 shows most vinculin located within the cytosol, not within the dense body. However the animals are not Pat, which suggests that some vinculin is present within the dense body as deb-1/vinculin null animals are Pat (Barstead and Waterston, 1991). In addition to their role as attachment proteins, all three LIM proteins are found within the nucleus throughout muscle development and growth (Figure 6). Whether any of these proteins shuttle between the nucleus and the plasma membrane as demonstrated for zyxin (Nix et al., 2001) has not been tested. Their localization to both the nucleus and the cytoplasm does make them candidate biosensors (Kadrmas and Beckerle, 2004).

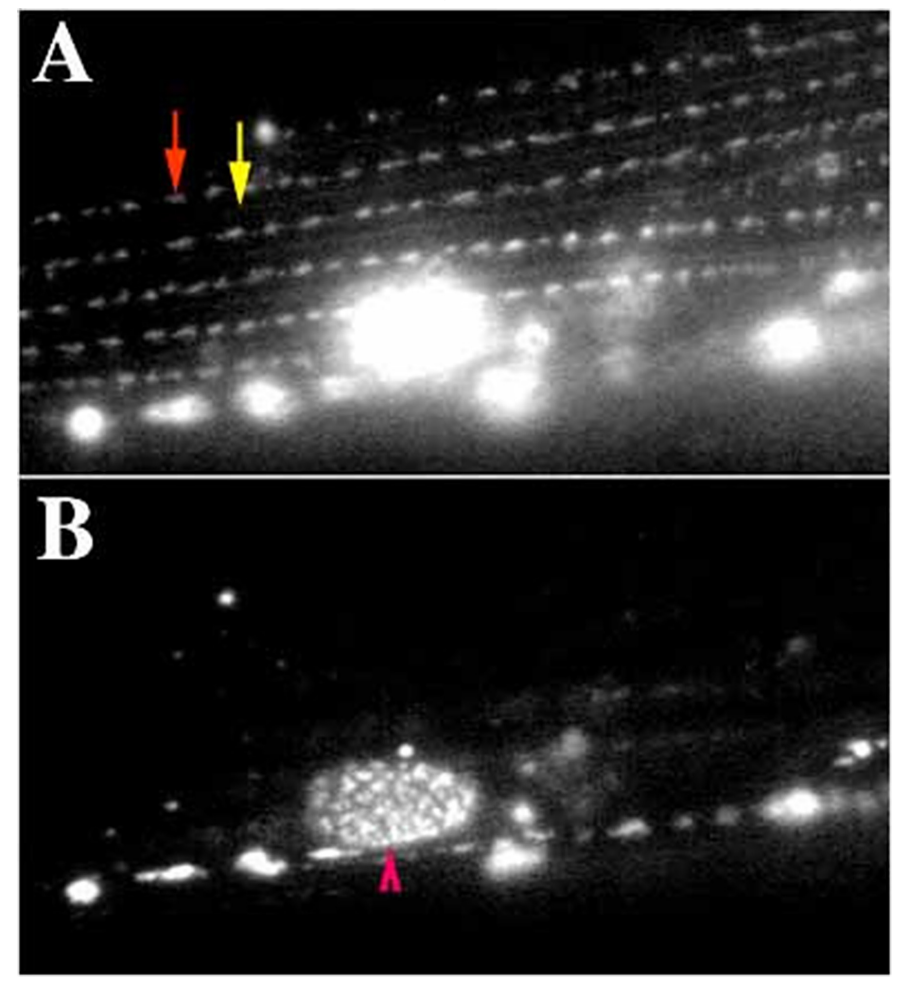

Figure 6. Localization of UNC-97::GFP within muscle body wall muscle. (A) UNC-97 is found in dense bodies (red arrow) and M-lines (yellow arrow) in mature muscle. (B) Within the same cells UNC-97 is found in a punctate or speckled pattern within the nucleus (red arrowhead). 
Figure 2, Figure 3, and Figure 5 suggest strong parallels between vertebrate and nematode attachment complexes, but there are some differences. The binding of the complex of talin, vinculin and alpha-actinin to integrin in vertebrates is through the FERM domain of talin (reviewed in Calderwood, 2004; Campbell and Ginsberg, 2004). By analogy we suggest that $C$. elegans talin does the same. However, the PAT-4/ILK complex behaves differently in vertebrates and C. elegans. In vertebrates ILK is the linker to integrin (Hannibal et al., 1996), but in C. elegans and Drosophila it is not (Mackinnon et al., 2002; Zervas et al., 2001). We suggest that binding of this complex to integrin may be mediated through UNC-112 since this protein when used as bait in a yeast two-hybrid assay identified PAT-3 integrin as a binding partner (Hiroshi Qadota and DGM, unpublished results). No follow-up binding studies have yet been done to confirm these findings but we find it intriguing that UNC-112, similar to talin, has a FERM domain that may bind integrin.

The assembly pathway described here relies on epistasis analysis, which would not be possible without mutants in each of the key molecules involved. The subset of Pat mutants described above may offer an entry point into the spatial control of adhesion sites. ILK and its binding partners do not appear to interfere with the formation of the "classical" talin vinculin adhesion complex, but removal of any member of the ILK complex inhibits the formation of a functional sarcomere. Myosin and actin filament networks are not assembled into sarcomeres, but even earlier, correct organization of integrin within the membrane is perturbed. Perhaps the role of the ILK complex, including UNC-112, UNC-97 and PAT-6, is to give definition to integrin spacing within the plane of the muscle plasma membrane. This spatial organization of integrin clusters may be achieved by linking the integrins to the underlying cytoskeleton which then "tows" the complex into position. Obviously, this is still a working hypothesis and needs to be verified through further experiments.

\section{Distinguishing dense bodies from M-lines}

The pathway described above gives an order of events starting at the cell membrane and progressing deeper into the cell. At some point along this progression adhesion plaques are segregated into two pools, one destined to be dense bodies and the other destined to be M-lines. These nascent adhesion plaques have at least nine proteins in common including integrin, talin, the ILK complex and the various LIM proteins. Divergence into distinct actin and myosin filament anchorage sites occurs after the addition of the ILK-binding complex, but presumably before alpha-actinin is added to the dense body or UNC-89 is added to the M-line. It is noteworthy in this regard that null mutations in alpha-actinin or unc-89 do not lead to a Pat phenotype (R. Barstead, personal commun; Benian et al., 1996). In fact, alpha-actinin is not even present at this stage of embryogenesis. Vinculin (possibly along with UNC-95) is the first component that is unique to the dense body over the M-line and is the first protein that allows us to distinguish these two adhesion complexes. Is it the addition of vinculin that gives an adhesion complex its specific identity as a dense body, and is an adhesion complex without vinculin by default an M-line? Consider a model in which vinculin is the key factor capable of determining whether a developing complex becomes a dense body or an M-line. The key issue would seem to be controlling the binding of vinculin to talin, perhaps by regulating the accessibility of binding sites on these two molecules (Izard et al., 2004; Fillingham et al., 2005). As a mechanism phosphorylation is one possibility that comes to mind, but one is then still faced with the problem of how such a switch distinguishes the adhesion complex pools. More specifically, how might such a switch be controlled spatially to create the characteristic, regular striated pattern of dense bodies and M-lines?

One idea that we explore further below in a speculative model is the possibility that the binary switch described above is simply stochastic. In other words, the initial isotropic fields of immature attachment complexes are randomly assigned either a dense body or M-line identity. How then is the ordered spatial array of dense bodies and M-lines established? The pattern could be established through the subsequent "pruning" of complexes that do not make productive transmembrane linkages. In our model a "productive" linkage is put under significant mechanical tension, which stabilizes it. In this view, nascent complexes formed "in the wrong place" fail and are disassembled, which frees their components to contribute to the continued growth of the appropriately positioned dense bodies and M-lines. The binary switch is not used to produce the pattern, but instead, patterning occurs after the switch has determined dense body/M-line fate. Below we speculate on mechanisms that might establish the patterned array of dense bodies and M-lines.

\section{Spacing of the components}

While it may seem counter-intuitive, adhesion plaques are relatively dynamic structures within tissue culture cells since they are not only important for adherence but are also necessary for cell motility. During nematode body wall muscle development integrin clusters must also have some flexibility. This can be observed during muscle cell 
growth. A newly formed muscle cell is two sarcomeres wide and has filaments $5 \mu \mathrm{m}$ long. In an adult animal this same muscle cell will be 10 sarcomeres wide and have individual filaments as long as $10 \mu \mathrm{m}$ (Waterston and Francis, 1985). Muscle cell mass increases greatly from an L1 lava to an adult so that individual dense bodies and M lines must also increase in size. A single A-band in an L1 larva has 100 filaments while an adult A-band may have as many as 600 filaments (MacKenzie et al., 1978). Increased filament length implies increased spacing between integrin clusters, and increased filament number implies larger integrin clusters. This latter point is important if the dense body is to increase in size, which it does. There is much in the literature on integrin clustering and on what some of the control elements may be (reviewed in Giancotti and Ruoslahti, 1999) and there are inroads being made to explain mechanosensitivity of integrin sites and the dynamics of integrin turnover during cell migration (Bershadsky et al., 2003; Wehrle-Haller and Imhof, 2002). However, with the notable exception of Ingber's theories on tensegrity (Ingber, 1997), there is little information available to explain how proper spacing of integrin within the plane of the membrane might be achieved.

Our model for the initial patterning of integrin into a proper array of dense bodies and M-lines relies on tissue interaction between muscle and hypodermis, and requires tension produced by the developing myofilament lattice. Several lines of evidence suggest that cytoskeletal patterning in muscle and hypodermis are tightly coupled during sarcomere assembly. The first suggestive evidence comes from the observation that in adults the muscle adhesion complexes and fibrous organelles (FO's) of the hypodermis, while not precisely aligned, do exhibit a tantalizing similarity in spacing. Strong evidence that muscle may induce the underlying patterning of the hypodermis comes from laser ablation experiments. Laser ablation of muscle precursors in the developing embryo leave substantial gaps in the normally continuous muscle quadrants. Underneath the muscle gaps there is no perlecan in the basement membrane (Moerman et al., 1996) and FO's fail to form in the underlying hypodermis (Hresko et al., 1999). One interpretation of these observations is that body wall muscle cells provide a signal that positions FO's in the hypodermis, and perhaps also induces FO assembly at appropriate sub cellular sites. Genetic analysis demonstrates that the hypodermal transmembrane protein myotactin is a key component in this tissue interaction. In myotactin loss-of-function mutants the FO's spread out laterally (and abnormally) into regions of hypodermis that are not in contact with body wall muscle (Hresko et al., 1999). What is particularly fascinating about myotactin is how it changes localization during the time of sarcomere assembly within body wall muscle. Initially myotactin is arranged in a pattern coinciding with the arrays of newly formed dense body and M-lines in the attached muscle cell just across the basement membrane, but at later stages of development myotactin moves from these nearly longitudinal lines into a series of circumferential lines as it joins the hypodermal FO's (Hresko et al., 1999). Myotactin may therefore be initially patterned by the muscle cell cytoskeleton.

There is also evidence that the hypodermis may influence muscle development and sarcomere assembly. Embryonic elongation is driven by circumferential actin cables assembled within the hypodermis (Priess and Hirsh, 1986). Mutations in the only alpha spectrin gene in C. elegans, spc-1, disrupts the regular patterning of these cables, causing the mutant embryos to elongate more slowly than normal, and eventually arrest at the two-fold length (Norman and Moerman, 2002). Interestingly, the A-bands in the body wall muscles of these arrested spc- 1 embryos are much wider than normal, and are oriented at $\sim 20$ degrees to the longitudinal access instead of the normal $\sim 6$ degrees. This wider zone within muscle for myofilament organization correlates with wider than normal zones of both UNC-52 depositions in the basement membrane and FO organization in the hypodermis. These changes in the muscle cytoskeleton are not simply due to a failure to complete elongation as several Pat mutants limited to expression in muscle (e.g., egl-19) exhibit normal myofilament orientation and A-band width (Norman and Moerman, 2002). These effects on the $s p c-1$ mutant muscle cells are also not simply caused by the absence of alpha spectrin in the muscle cells. The unc-70 gene encodes beta-spectrin (Hammerlund et al., 2000), and in unc-70 mutants, alpha-spectrin remains cytoplasmic in body wall muscle cells, instead of localizing normally to the muscle cell membrane. The mutant unc-70 embryos have normal muscle structure, suggesting that it is the loss of spectrin activity in the hypodermis that is altering the patterning of muscle sarcomeres (Norman and Moerman, 2002). Although the mechanism responsible for this effect remains to be elucidated, these results raise the interesting possibility that the contracting hypodermis affects the amount of muscle cell surface area that comes into contact with the hypodermis during the initial assembly of sarcomeres. This change in the basal surface of the muscle cell in turn has a direct effect on the spatial orientation of the incipient sarcomeres, the width of the basement membrane and, by extension, the width of FO's within the hypodermis.

As well as hypodermal/muscle contact there is evidence that mechanical tension is needed for the proper formation of muscle-hypodermal cytoskeletal linkages. Body wall muscle cells never contract forcefully in Pat mutants, and it appears that the resulting lack of tension blocks the normal development muscle-hypodermal linkages. The myo-3 Pat mutants provide the best example. The myo-3 gene encodes myosin A protein, which is the 
central component of body wall muscle thick filaments (Waterston 1989). Despite the fact that myosin A is not a structural component of muscle attachment complexes, linkages to the hypodermis are extremely weak in these animals. This is shown by the frequent detachment of their dorsal muscle quadrants from the body wall near the bend of the arrested two fold embryos. These detachments are a characteristic that is shared by a subset of Pat embryos able to weakly contract their body wall muscle cells . In the myo-3 mutants, we speculate that these weak movements are powered by other myosin isoforms expressed by the muscle cells.

Our model for how sarcomere spacing may be achieved is as follows. UNC-52/perlecan begins to accumulate in the extracellular space separating two longitudinal rows of muscle cells making up each quadrant (green, Figure $7 \mathrm{~A}$ ), and then initiates the assembly of muscle attachments in the basal membrane adjacent the centerline of the quadrant. The adhesions first appear as two parallel stripes closely flanking the quadrant centerline (black dots, Figure 7A, also Figure 7E-G). The newly formed complexes are small and are randomly positioned within each of the stripes. Actin filaments organize next through their association with the attachment complexes, resulting in two large, longitudinally oriented bundles of actin filaments flanking the quadrant centerline (vertical red lines; Figure 7B; Figure 8). Myosin assembles into thick filaments and then forms larger aggregates (blue lines; Figure 7B-C) that are dispersed throughout the muscle cell cytoplasm (see Epstein et al., 1993). The myosin aggregates interact with the bundled actin filaments, and are also recruited to the centerline. Actin and myosin filaments begin to interdigitate, and the resulting myofilament lattice begins to contract (Figure 7C). During the next phase of muscle development a subset of the attachment complexes continue to grow and form longitudinal rows of uniformly spaced dense bodies, while others are either disassembled or are repositioned to join the successful attachments. For a dense body to persist during this phase it must be under significant tension from the myofilament lattice and must also become mechanically linked to the actin cables in the adjacent hypodermis (Figure 7G). This process is illustrated at the bottom of Figure 7B-D, which shows the loss of small integrin clusters from sites not immediately adjacent to actin cables in the underlying hypodermis (black horizontal lines), and the simultaneous enlargement of the remaining muscle-muscle attachment complexes (black half-circles; Figure 7D). These combined constraints limit the locations where dense bodies can mature, and ultimately imposes the longitudinal spacing between these mature attachments. The M-line complexes require only linkage to the center of myosin thick filaments to persist, which permits the very close spacing that often appears as a continuous line (see Figure 7H-J). It is important to note that because each muscle cell is two sarcomeres "wide", one end of every sarcomere is precisely positioned by the intersecting "grid" of nearly longitudinal muscle-muscle junctions and the circumferential hypodermal actin cables (Figure 7D). Thus, it may be this intersection that initially determines the spacing of dense bodies and M-lines. In Figure 7G, we diagram a hypothetical "linker" protein that binds UNC-52, which in turn may be bound by integrin, thus establishing a mechanical linkage that stabilizes the nascent dense body or muscle attachment plaque.

The idea that actin cytoskeleton in the hypodermis might establish the initial spacing of dense bodies is only one of many possible mechanisms that might bring this about. We raise this idea because the muscle and hypodermis are clearly interacting in important ways at this stage of development, and it is intriguing that the spacing between actin cables and newly formed dense bodies is of the same order of magnitude. It should be noted, however, that we are not aware of any data demonstrating that the position of actin cables and newly forming muscle attachments coincides precisely, as this model predicts and is indicated in Figure 7. 


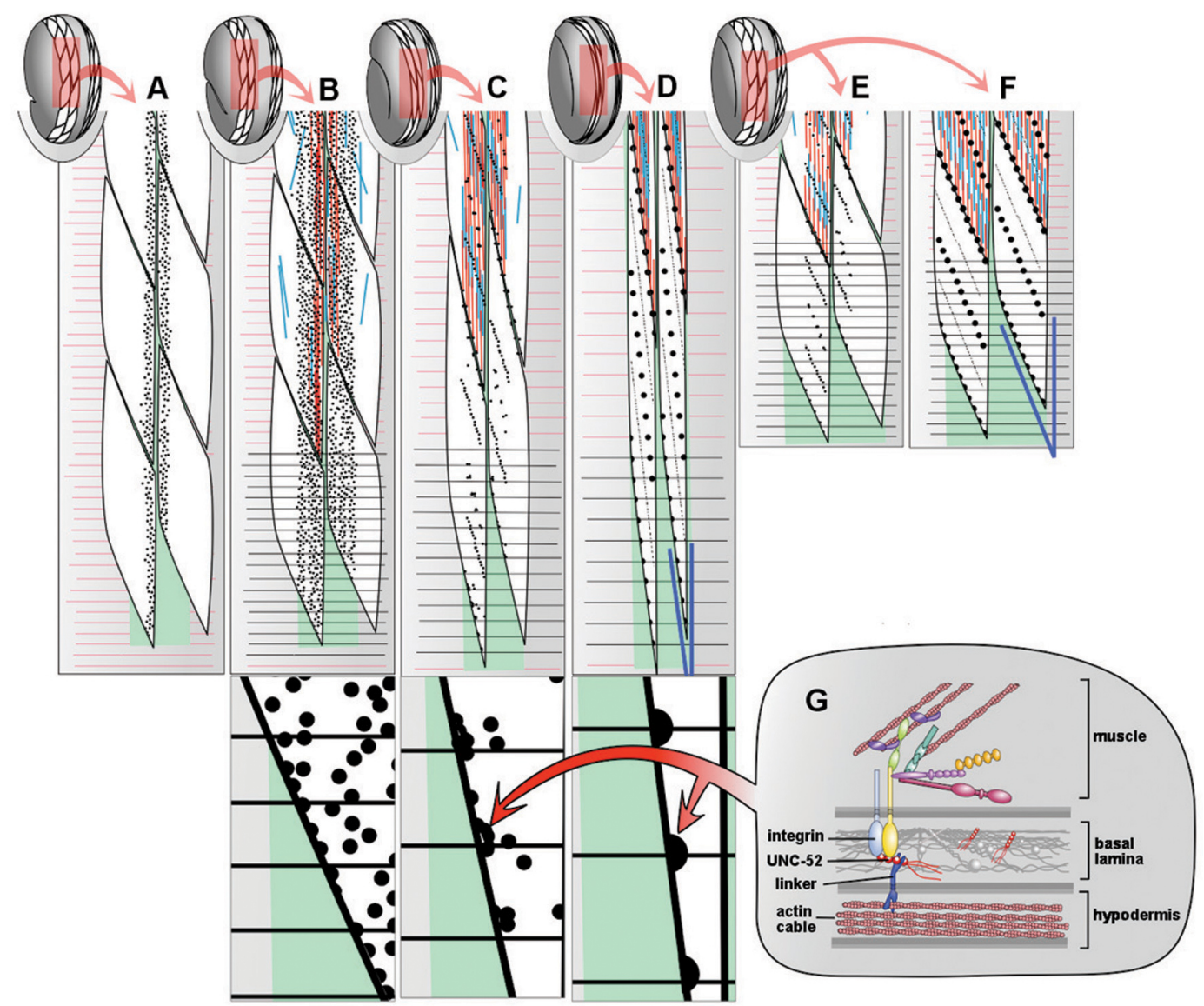

Figure 7. Model of sarcomere development showing several adjacent cells in one dorsal muscle quadrant at several successive developmental stages. Developmental stage is indicated by the extent of elongation in embryo cartoons at the top. Wild-type (A-D) and $s p c-1$ mutant embryos (E, F) are shown. In panels A-F, muscle cells (white) are shown on top of the basal lamina (light green) and the basal face of the hypodermis (shaded gray). The bottom of panels B-D show enlarged views of developing muscle attachments (black dots), with the position of hypodermal circumferential actin cables indicated (black horizontal red lines). G shows a hypothetical linkage between muscle attachments and hypodermal actin cables involving a "linker protein" bound to UNC-52/perlecan, which in turn binds integrin. Note that direct binding between integrin and UNC-52 has not been demonstrated in $C$. elegans, although integrin-perlecan binding has been demonstrated in vertebrates. See text for further details.

Why are the dense body rows pitched $\sim 6$ degrees from the longitudinal axis? We propose that the angle is imposed by shape of the muscle cells at the time when dense bodies and the related adhesion plaques (hemi-dense bodies) become stabilized by linkage to the hypodermal cytoskeleton. The muscle cells have assumed a spindle shape by the one-and-three-quarter-fold stage, and muscle-muscle junctions between anterior-posterior neighbors are approximately 6 degrees from longitudinal. The intersections between these junctions and the nearly orthogonal pattern of actin hypodermal actin cables define a series of points where attachment plaques will persist and mature (unipolar dense bodies). Interestingly, in the spc-1 mutants elongation is much slower than normal, so dense bodies and attachment plaques are "selected" when the muscle cells are in the "wrong" shape. The cells are much wider than they should be for their chronological age, and the muscle-muscle junctions between anterior-posterior neighbors are closer to 20 than to 6 degrees (Figure 7E-F). The A-bands, like the muscle cells, are too wide, and their angle to the longitudinal axis is 20 degrees. 


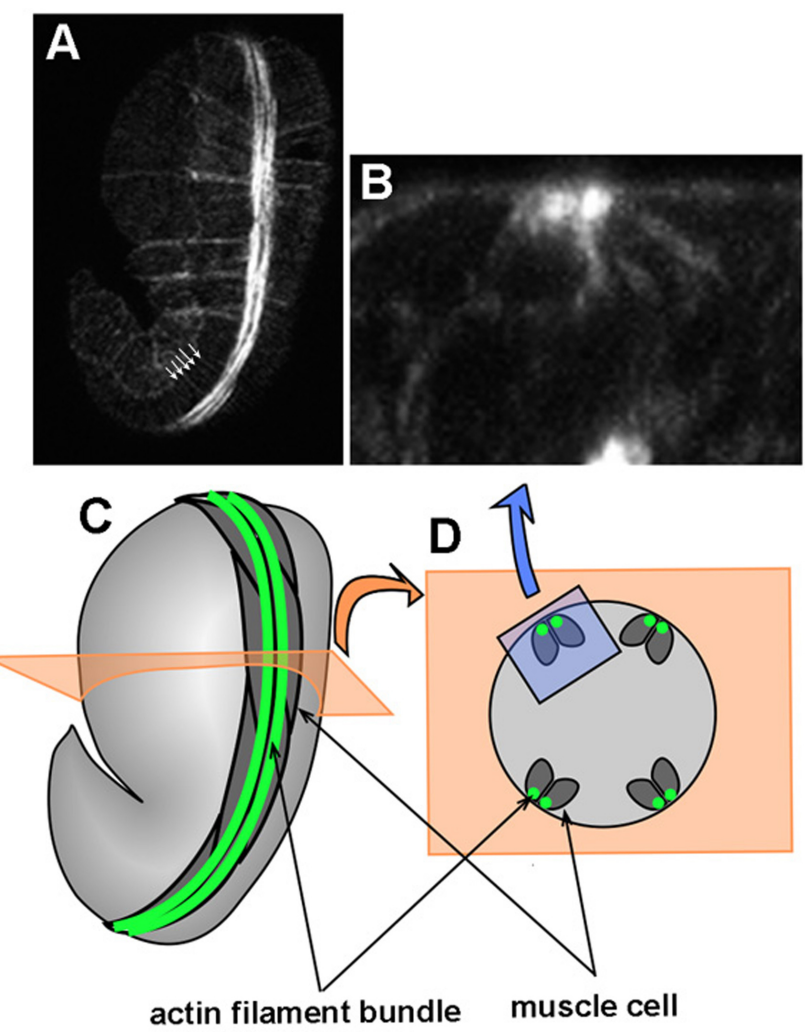

Figure 8. View of a phalloidin stained 1.5 fold wild type embryo. (A) Whole embryo shown from a left dorsal view. Two bundles of actin filaments running longitudinally along left dorsal muscle quadrant are visible. The outline of hypodermal cells is also visible as well the circumferential actin cables (arrows). (B) A cross section of the embryo illustrating the polarized position of the actin filaments within the muscle cells. C and D are schematic views of $\mathrm{A}$ and $\mathrm{B}$ respectively.

\section{Is sarcomere assembly in C. elegans a general model of sarcomere assembly?}

A key question is whether these studies on sarcomere assembly in C. elegans have any relevance for mammalian muscle development. For several years those working on myogenesis in vertebrates largely ignored the work on $C$. elegans sarcomere assembly. At the same time those working on adhesion complexes have used observations in C. elegans and Drosophila melanogaster to advance our understanding of adhesion complexes in mammals (reviewed in Labouesse and Georges-Labouesse, 2003). Three examples serve to illustrate this point. First, recent studies on flies and worms demonstrate that ILK is an adaptor molecule and all of its behavior can be explained as an adaptor molecule, not as a kinase (Zervas et al., 2001; Mackinnon et al., 2002; reviewed in Zervas and Brown, 2002). These observations have been confirmed in mammals using an ILK knockout mouse (Sakai et al., 2003). Second, it was the genetic analysis in C. elegans that first demonstrated the key roles of UNC-112, UNC-97, PAT-4 and PAT-6 in the assembly of integrin attachment complexes. Third, the observation that UNC-112/Mig-2 is localized to adhesion complexes, and binds ILK and PINCH, led Tu et al. (2003) to recapitulate these results and do 2-hybrid screens for a novel Mig-2 binding partner. This led to the isolation of a protein with a proline rich amino terminus and three LIM domains, Migfilin, which they demonstrated binds to filamin. This is the first example linking filamin regulation of actin filaments to the integrin/vinculin pathway for actin filament assembly. Finally, and to come full circle, the critical role played by beta 1 integrin in early mouse myogenesis has recently been demonstrated. In a mouse mutant lacking beta 1 integrin, recruitment of talin and vinculin to costamers is inhibited and proper rcomeres are not formed (Schwander et al., 2003). The phenotype observed is reminiscent of Pat mutants in C. elegans confirming the phylogenetic conservation of integrin complexes in sarcomere assembly.

\section{Summary}

We, and others, have made significant inroads into how a muscle sarcomere is initiated and some progress on dissecting the assembly pathway that leads to this complex structure. However, there are several outstanding issues that remain including " 
1. How does the cell distinguish adhesion sites destined to be dense bodies from those destined to be M-lines?

2. How is the precise spacing between sarcomere units generated and maintained throughout development?

3. How are actin and myosin microfilaments inserted into the nascent sarcomere, and then how is filament length regulated as the animal grows?

These are difficult questions, ones that we think go to the heart of a very difficult problem in cytoarchitecture. C. elegans may offer the best opportunity to solve these challenging problems in macromolecular assembly and cellular organization.

\section{Acknowledgements}

We thank Ken Norman for Figure 8 and Hiroshi Qadota for permission to cite his data on UNC-112 binding to integrin. This review benefited greatly from the insightful comments of an anonymous reviewer. Research in the laboratory of DGM relevant to this review was supported by grants from the Canadian Institute for Health Research, the National Science and Engineering Research Council of Canada, the Heart and Stroke Foundation of Canada and the Health Research Foundation of British Columbia. Research in the laboratory of BDW relevant to this review was supported by grants from the National Institutes of Health (R01 HD38646) and the American Heart Association, Midwest Affiliate.

\section{References}

Barstead, R.J., and Waterston, R.H. (1991). Cloning, sequencing and mapping of an alpha-actinin gene from the nematode Caenorhabditis elegans. Cell Motil. Cytoskeleton 20, 69-78. Abstract Article

Barstead, R.J., and Waterston, R.H. (1991). Vinculin is essential for muscle function in the nematode. J. Cell Biol. 114, 715-724. Abstract Article

Benian, G.M., Tinley, T.L., Tang, X., and Borodovsky, M. (1996). The Caenorhabditis elegans gene unc-89, required fpr muscle M-line assembly, encodes a giant modular protein composed of Ig and signal transduction domains. J. Cell Biol. 132, 835-848. Abstract Article

Bercher, M., Wahl, J., Vogel, B.E., Lu, C., Hedgecock, E.M., Hall, D.H., and Plenefisch, J.D. (2001). mиa-3, a gene required for mechanical tissue integrity in Caenorhabditis elegans, encodes a novel transmembrane protein of epithelial attachment complexes. J. Cell Biol. 154, 415-426. Abstract Article

Bershadsky, A.D., Balaban, N.Q., and Geiger, B. (2003). Adhesion-dependent cell mechnosensitivity. Annu. Rev. Cell Dev. Biol. 19, 677-695. Abstract Article

Bosher, J.M., Hahn, B.S., Legouis, R., Sookhareea, S., Weimer, R.M., Gansmuller, A., Chisholm, A.D., Rose, A.M., Bessereau, J.L., and Labouesse, M. (2003). The Caenorhabditis elegans vab-10 spectraplakin isoforms protect the epidermis against internal and external forces. J. Cell Biol. 161, 757-768. Abstract Article

Broday, L., Kolotuev, I., Didier, C., Bhoumik, A., Podbilewicz, B., and Ronai, Z. (2004). The LIM domain protein UNC-95 is required for the assembly of muscle attachment structures and is regulated by the RING finger protein RNF-5 in C. elegans. J. Cell Biol. 165, 857-867. Abstract Article

Brown, J.C., Sasaki, T., Gohring, W., Yamada, Y., and Timpl, R. (1997). The C-terminal domain V of perlecan promotes beta1 integrin-mediated cell adhesion, binds heparin, nidogen and fibulin-2 and can be modified by glycosaminoglycans. Eur. J. Biochem. 250, 39-46. Abstract Article

Burridge, K., and Chrzanowska-Wodnicka, M. (1996). Focal adhesions, contractility, and signaling. Annu. Rev. Cell Dev. Biol. 12, 463-518. Abstract Article

Calderwood, D.A. (2004). Taliin controls integrin activation. Biochem. Sci. Transactions 32(pt3), 434-437. Article 
Campbell, I.D., and Ginsberg, M.H. (2004). The talin-tail interaction places integrin activation on FERM ground. Trends Biochemcal Sciences 8, 429-435. Article

Chakravarti, S., Horchar, T., Jefferson, B., Laurie, W., and Hassell, J.R. (1995). Recombinant domain III of perlecan promotes cell attachment through its RGDS sequence. J. Biol. Chem. 270, 404-409. Abstract Article

Cox, E.A., and Hardin, J. (2004). Sticky worms: adhesion coomplexes in Caenorhabditis elegans. J. Cell Sci. 117, 1885-1897. Abstract Article

Ding, M., Goncharov, A., Jin, Y., and Chisholm, A.D. (2003). C. elegans ankyrin repeat protein VAB-19 is a component of epidermal attachment structures and is essential for epidermal morphogenesis. Development 130, 5791-5801. Abstract Article

Epstein, H.F., Casey, D.L., and Ortiz, I. (1993). Myosin and paramyosin of Caenorhabditis elegans embryos assemble into nascent structures distinct from thick filaments and multi-filament assemblages. J. Cell Biol. 122, 845-858. Abstract Article

Fillingham, I., Gingras, A.R., Papagrigoriou, E., Patel, B., Emsley, J., Critchley, D.R., Roberts, G.C., and Barsukov, I.L. (2005). A vinculin binding domain from the talin rod unfolds to form a complex with the vinculin head. Structure (Camb.) 13, 65-74. Article

Francis, G.R., and Waterston, R.H. (1985). Muscle organization in Caenorhabditis elegans: localization of proteins implicated in thin filament attachment and I-band organization. J. Cell Biol. 101, 1532-1549. Abstract Article

Francis, R., and Waterston, R.H. (1991). Muscle cell attachment in Caenorhabditis elegans. J. Cell Biol. 114, 465-479. Abstract Article

Geiger, B., and Bershadsky, A. (2001). Assembly and mechanosensory function of focal contacts. Curr. Opin. Cell Biol. 13, 584-592. Abstract Article

Gettner, S.N., Kenyon, C., and Reichardt, L.F. (1995). Characterization of beta pat-3 heterodimers, a family of essential integrin receptors in C. elegans. J. Cell Biol. 129, 1127-1141. Abstract Article

Giancotti, F.G., and Ruoslahti, E. (1999). Integrin signaling. Science 285, 1028-1032. Abstract Article

Hammerlund, M., Davis, W.S., and Jorgensen, E.M. (2000). Mutations in Beta-spectrin disrupt axon outgrowth and muscle attachment structures. J. Cell Biol. 149, 931-942. Article

Hannigan, G.E., Leung-Hagesteijn, C., Fitz-Gibbon, L., Coppolino, M.G., Radeva, G., Filmus, J., Bell, J.C., and Dedhar, S. (1996). Regulation of cell adhesion and anchorage-dependent growth by a new beta 1-integrin-linked protein kinase. Nature 379, 91-96. Abstract Article

Hapiak, V., Hresko, M.C., Schriefer, L.A., Saiyasisongkhram, K., Bercher, M., and Plenefisch, J. (2003). mиа-6, a gene required for tissue integrity in Caenorhabditis elegans, encodes a cytoplasmic intermediate filament. Dev. Biol. 263, 330-342. Abstract Article

Hayashi, K., Madri, J.A., and Yurchenco, P.D. (1992). Endothelial cells interact with the core protein of basement membrane pelecan through beta 1 and beta 3 integrins: an adhesion modulated by glycosaminoglycan. J. Cell Biol. 119, 945-959. Abstract Article

Hobert, O., Moerman, D.G., Clark, K.A., Beckerle, M.C., and Ruvkun, G. (1999). A conserved LIM protein that affects muscular adherens junction integrity and mechanosensory function in Caenorhabditis elegans. J. Cell Biol. 144, 45-57. Abstract Article

Hong, L., Elbl, T., Ward, J., Franzini-Armstrong, C., Rybicka, K.K., Gatewood, B.K., Baillie, D.L., and Bucher, E.A. (2001). MUP-4 is a novel transmembrane protein with functions in epithelial cell adhesion in Caenorhabditis elegans. J. Cell Biol. 154, 403-414. Abstract Article

Hresko, M.C., Schriefer, L.A., Shrimankar, P., and Waterston, R.H. (1999). Myotactin, a novel hypodermal protein involved in muscle-cell adhesion in Caenorhabditis elegans. J. Cell Biol. 146, 659-672. Abstract Article 
Hresko, M.C., Williams, B.D., and Waterston, R.H. (1994). Assembly of body wall muscle and muscle cell attachment structures in Caenorhabditis elegans. J. Cell Biol. 124, 491-506. Abstract Article

Ingber, D.E. (1997). Tensegrity: the architectural basis of cellular mechanotransduction. Annu. Rev. Physiol. 59, 575-599. Abstract Article

Izard, T., Evans, G., Borgon, R.A., Rush, C.L., Bricogne, G., and Bois, P.R. (2004). Vinculin activation by talin through helical bundle conversion. Nature 427, 171-175. Abstract Article

Kadrmas, J.L., and Beckerle, M.C. (2004). The LIM domain: from the cytoskeleton to the nucleus. Nat. Rev. Mol. Cell Biol. 5, 920-931. Abstract Article

Labouesse, M., and Georges-Labouesse, E. (2003). Cell adhesion: parallels between vertebrate and invertebrate focal adhesions. Curr. Biol. 13, R528-R530. Abstract Article

Lin, X., Qadota, H., Moerman, D.G., and Williams, B.D. (2003). C. elegans PAT-6/actopaxin plays a critical role in the assembly of integrin adhesion complexes in vivo. Curr. Biol. 13, 922-932. Abstract Article

Mackenzie, J.M., Jr., Garcea, R.L., Zengel, J.M., and Epstein, H.F. (1978). Muscle development in Caenorhabditis elegans: mutants exhibiting retarded sarcomere construction. Cell 15, 751-762. Abstract Article

Mackinnon, A.C., Qadota, H., Norman, K.R., Moerman, D.G., and Williams, B.D. (2002). C. elegans PAT-4/ILK functions as an adaptor protein within integrin adhesion complexes. Curr. Biol. 12, 787-797. Abstract Article

Mercer, K.B., Flaherty, D.B., Miller, R.K., Qadota, H., Tinley, T.L., Moerman, D.G., and Benian, G.M. (2003). Caenorhabditis elegans UNC-98, a C2H2 Zn finger protein, is a novel partner of UNC-97/PINCH in muscle adhesion complexes. Mol. Biol. Cell 14, 2492-2507. Abstract Article

Moerman, D.G., and Fire, A. (1997). Muscle: Structure, function and development. In C. elegans II, D.L. Riddle, T. Blumenthal, B.J. Meyer, and J.R. Priess, eds. (Cold Spring Harbor: Cold Spring Harbor Press), pp. 417-470.

Moerman, D.G., Hutter, H., Mullen, G.P., and Schnabel, R. (1996). Cell autonomous expression of perlecan and plasticity of cell shape in embryonic muscle of Caenorhabditis elegans. Dev. Biol. 173, 228-242. Abstract Article

Moulder, G.L., Huang, M.M., Waterston, R.H., and Barstead, R.J. (1996). Talin requires beta-integrin, but not vinculin, for its assembly into focal adhesion-like structures in the nematode Caenorhabditis elegans. Mol. Biol. Cell 7, 1181-1193. Abstract

Mullen, G.P., Rogalski, T.M., Bush, J.A., Gorji, P.R., and Moerman, D.G. (1999). Complex patterns of alternative splicing mediate the spatial and temporal distribution of perlecan/UNC-52 in Caenorhabditis elegans. Mol. Biol. Cell 10,3205-3221. Abstract

Nix, D.A., Fradelizi, J., Bockholt, S., Menichi, B., Louvard, D., Friederich, E., and Beckerle, M.C. (2001). Targeting of zyxin to sites of actin membrane interaction and to the nucleus. J. Biol. Chem. 276, 34759-34767. Abstract Article

Norman, K.R., and Moerman, D.G. (2002). Alpha spectrin is essential for morphogenesis and body wall muscle formation in Caenorhabditis elegans. J. Cell Biol. 157, 665-677. Abstract Article

Priess, J.R., and Hirsh, D. (1986). Caenorhabditis elegans morphogenesis: The role of the cytoskeleton in the elongation of the embryo. Dev. Biol. 117, 156-173. Abstract Article

Rogalski, T.M., Mullen, G.P., Gilbert, M.M., Williams, B.D., and Moerman, D.G. (2000). The UNC-112 gene in Caenorhabditis elegans encodes a novel component of cell-matrix adhesion structures required for integrin localization in the muscle cell membrane. J. Cell Biol. 150, 253-264. Abstract Article

Rogalski, T.M., Williams, B.D., Mullen, G.P., and Moerman, D.G. (1993). Products of the unc-52 gene in Caenorhabditis elegans are homologous to the core protein of the mammalian basement membrane heparan sulfate proteoglycan. Genes Dev. 7, 1471-1484. Abstract 
Sakai, T., Li, S., Docheva, D., Grashoff, C., Sakai, K., Kostka, G., Braun, A., Pfeifer, A., Yurchenco, P.D., and Fassler, R. (2003). Integrin-linked kinase (ILK) is required for polarizing the epiblast, cell adhesion, and controlling actin accumulation. Genes Dev. 17, 926-940. Abstract Article

Schnabel, R., Hutter, H., Moerman, D., and Schnabel, H. (1997). Assessing normal embryogenesis in Caenorhabditis elegans using a 4D microscope: variability of development and regional specification. Dev. Biol. 184, 234-265. Abstract Article

Schwander, M., Leu, M., Stumm, M., Dorchies, O.M., Ruegg, U.T., Schittny, J., and Muller, U. (2003). Beta1 integrins regulate myoblast fusion and sarcomere assembly. Dev. Cell 4, 673-685. Abstract Article

Sulston, J.E., Schierenberg, E., White, J.G., Thomson, J.N. (1983). The embryonic cell lineage of the nematode Caenorhabditis elegans. Dev Biol. 100, 64-119. Abstract

Tu, Y., Li, F., Goicoechea, S., and Wu, C. (1999). The LIM-only protein PINCH directly interacts with integrin-linked kinase and is recruited to integrin-rich sites in spreading cells. Mol. Cell Biol. 19, 2425-2434. Abstract

Tu, Y., Wu, S., Shi, X., Chen, K., and Wu, C. (2003). Migfilin and Mig-2 link focal adhesions to filamin and the actin cytoskeleton and function in cell shape modulation. Cell 113,37-47. Abstract Article

Waterston, R.H. (1988). Muscle. In The nematode Caenorhabditis elegans, W.B. Wood, ed. (Cold Spring Harbor: Cold Spring Harbor Press), pp. 281-335.

Waterston, R.H. (1989). The minor myosin heavy chain gene, MHC A, of Caenorhabditis elegans is necessary for the intitiation of thick filament assembly. EMBO J. 8, 3429-3436. Abstract

Waterston, R.H., and Francis, G.R. (1985). Genetic analuysis of muscle development in Caenorhabditis elegans. Trends Neurosci. 84, 270-276. Article

Wehrle-Haller, B., and Imhof, B.A. (2002). The inner lives of focal adhesions. Trends Cell Biol. 12, 382-389. Abstract Article

Williams, B.D., and Waterston, R.H. (1994). Genes critical for muscle development and function in Caenorhabditis elegans identified through lethal mutations. J. Cell Biol. 124, 475-490. Abstract Article

Zervas, C.G., and Brown, N.H. (2002). Integrin adhesion: when is a kinase a kinase? Curr. Biol. 12, R350-R351. Abstract Article

Zervas, C.G., Gregory, S.L., and Brown, N.H. (2001). Drosophila integrin-linked kinase is required at sites of integrin adhesion to link the cytoskeleton to the plasma membrane. J. Cell Biol. 152, 1007-1018. Abstract Article

All WormBook content, except where otherwise noted, is licensed under a Creative Commons Attribution License 